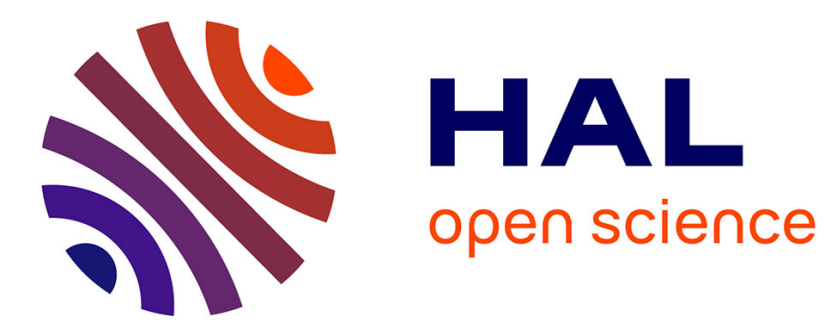

\title{
Relative motion of satellites exploiting the super-integrability of Kepler's problem
}

\author{
K. Uldall Kristiansen, P. L. Palmer, M. Roberts
}

\section{To cite this version:}

K. Uldall Kristiansen, P. L. Palmer, M. Roberts. Relative motion of satellites exploiting the superintegrability of Kepler's problem. Celestial Mechanics and Dynamical Astronomy, 2010, 106 (4), pp.371-390. 10.1007/s10569-009-9253-y . hal-00568374

\section{HAL Id: hal-00568374 https://hal.science/hal-00568374}

Submitted on 23 Feb 2011

HAL is a multi-disciplinary open access archive for the deposit and dissemination of scientific research documents, whether they are published or not. The documents may come from teaching and research institutions in France or abroad, or from public or private research centers.
L'archive ouverte pluridisciplinaire HAL, est destinée au dépôt et à la diffusion de documents scientifiques de niveau recherche, publiés ou non, émanant des établissements d'enseignement et de recherche français ou étrangers, des laboratoires publics ou privés. 


\title{
Relative Motion of Satellites Exploiting the Super-Integrability of Kepler's Problem
}

\author{
K. Uldall Kristiansen ${ }^{\dagger \ddagger}$, P.L. Palmer ${ }^{\dagger}$ and M. Roberts ${ }^{\ddagger}$ \\ Surrey Space Centre ${ }^{\dagger}$ and Department of Mathematics ${ }^{\ddagger}$, \\ University of Surrey, \\ Guildford, GU2 7XH, \\ UK
}

\begin{abstract}
This paper builds upon the work of Palmer and Imre exploring the relative motion of satellites on neighbouring Keplerian orbits. We make use of a general geometrical setting from Hamiltonian systems theory to obtain analytical solutions of the variational Kepler equations in an Earth centred inertial coordinate frame in terms of the relevant conserved quantities: relative energy, relative angular momentum and the relative eccentricity vector. The paper extends the work on relative satellite motion by providing solutions about any elliptic, parabolic or hyperbolic reference trajectory, including the zero angular momentum case. The geometrical framework assists the design of complex formation flying trajectories. This is demonstrated by the construction of a tetrahedral formation, described through the relevant conserved quantities, for which the satellites are on highly eccentric orbits around the sun to visit the Kuiper belt.
\end{abstract}




\section{Introduction}

The modelling and analysis of the relative motion of two satellites is of immediate relevance to the design of multiple satellite missions such as constellations and formations. Significant interest in this area is demonstrated by Xiang and Jørgensen's recent survey [26] of planned and existing formation flying missions. This lists as many as 15 current and future missions. Recent examples include the NASA EO-1 remote sensing mission $[6,8]$, the GRACE mission mapping the Earth's gravity field [20] and the CLUSTER II space weather and environment mission with four satellites in a tetrahedral formation [4].

Perhaps the best known relative motion model is Hill's equations [10], adapted to the problem of relative satellite navigation by Clohessy and Wiltshire [2] in 1960s. This is the linearisation of Keplerian relative dynamics around a circular reference orbit. However, six years prior to Clohessy and Wiltshire, Lawden derived the basic equations of relative motion for the more general case of eccentric orbits [13] (c.f. [1]). Tschauner and Hempel [22] independently formulated similar solutions to Lawden's around the same time. The approach is in fact a generalisation of the Clohessy-Wiltshire equations, solving the same problem but linearising around an eccentric orbit rather than a circular one. While they still use Hill's frame, they employ the true anomaly as the independent variable.

As formation flying missions are becoming a reality, there has been a renewed interest in relative motion modelling within the last decade. Important and recent references include $[1,15]$ for the linear theory, $[5,9,17,18,25]$ for inclusion of higher order geopotential terms and $[7,12,19,23]$ for nonlinearity effects. One of the few common threads within the existing literature on relative motion, is that virtually all of the methods use a rotating and accelerating local coordinate frame. This approach makes analysis and visualisation of the motion rather straightforward. However, the perturbations to the Keplerian potential are usually defined in the Earth Centred Inertial (ECI) frame, or sometimes the rotating Earth Centred Earth Fixed (ECEF) frame. This is one of the primary reasons why the addition

of the simple $J_{2}$ perturbation term greatly complicates the equations. The modelling of the 
motion is actually hampered by the employment of this accelerating rotating frame.

Perhaps more importantly, these methods do not explicitly address the issue of constants of motion. For the motion of a satellite under a Keplerian potential, the energy, the angular momentum and the eccentricity are all conserved. For the case of the two satellites; the 'relative energy', the 'relative angular momentum', and the 'relative eccentricity' are also conserved. If these quantities are not conserved, e.g. in numerical integration, the relative orbits will get distorted over time. For example, any deviation from the relative energy will manifest itself as an alongtrack drift.

Palmer and Imre $[11,16]$ incorporated conservation of relative energy and relative angular momentum. In [16] they solved the linearised relative motion in the ECI coordinate frame exploiting these quantities, and in [11] a symplectic numerical relative orbit propagator was developed imposing the conservation laws and incorporating high order geopotentials. The results demonstrated a $40 \%$ improvement in computational time compared with propagating separate orbits with a similar level of accuracy.

This paper extends the work by Palmer and Imre in [16] by providing a generalised mathematical foundation and using the conservation of relative energy, angular momentum and eccentricity to obtain solutions, expressed only in terms of these conserved quantities and a time delay. The Keplerian orbits are easily interpreted geometrically through conic sections and the conserved quantities and this framework provides the variational solutions with a natural, similar geometry. This geometry is useful in applications of formation flying mission design which we demonstrate by the construction of a tetrahedron formation. Furthermore, unlike the other work on the subject of relative motion mentioned above, we present a complete solution of the variational equations valid for all elliptic, hyperbolic and parabolic reference trajectories, including the zero angular momentum case. 


\section{The Kepler problem}

The two-body problem is given by the Hamiltonian

$$
H(\mathbf{q}, \mathbf{p})=\frac{1}{2}|\mathbf{p}|^{2}-\frac{\mu}{|\mathbf{q}|},
$$

on the manifold $M=T^{*} Q \ni(\mathbf{q}, \mathbf{p})$, where $Q=\mathbb{R}^{3} \backslash\{0\}$ is the configuration space, endowed with the symplectic form $\omega=\mathrm{d} \mathbf{q} \wedge \mathrm{d} \mathbf{p}$. Kepler's equations then coincide with Hamilton's equations:

$$
\begin{aligned}
& \dot{\mathbf{q}}=\partial_{\mathbf{p}} H(\mathbf{q}, \mathbf{p})=\mathbf{p} \\
& \dot{\mathbf{p}}=-\partial_{\mathbf{q}} H(\mathbf{q}, \mathbf{p})=-\frac{\mu}{q^{3}} \mathbf{q},
\end{aligned}
$$

where $q=|\mathbf{q}|,|\cdot|$ being the Euclidean norm. ${ }^{1}$ By the rotational invariance, $H(\mathbf{R q}, \mathbf{R} \mathbf{p})=$ $H(\mathbf{q}, \mathbf{p})$ for every rotation matrix $\mathbf{R}$, it follows that

$$
\mathbf{L}(\mathbf{q}, \mathbf{p})=\mathbf{q} \wedge \mathbf{p}
$$

is a conserved quantity. From the conservation of $\mathbf{L}$ follows that the motion takes place in a plane, which is called the orbital plane:

$$
P=\left\{\mathbf{x} \in \mathbb{R}^{3} \mid\langle\mathbf{x}, \mathbf{k}\rangle=0\right\}
$$

where $\mathbf{k}$ is a unit vector in the direction of $\mathbf{L}$. If $\mathbf{L}(\mathbf{q}, \mathbf{p})=\mathbf{0}$ then $\mathbf{q} \| \mathbf{p}$ and the motion is radial and may, depending on the energy, only exist for a finite time.

Due to a more hidden symmetry [3], the eccentricity vector

$$
\mathbf{e}(\mathbf{q}, \mathbf{p})=-\frac{\mathbf{q}}{|\mathbf{q}|}+\frac{1}{\mu} \mathbf{p} \wedge \mathbf{L}(\mathbf{q}, \mathbf{p})
$$

is also conserved.

The seven scalar conserved quantities $H(\mathbf{q}, \mathbf{p})=h, \mathbf{L}(\mathbf{q}, \mathbf{p})=\mathbf{l}$ and $\mathbf{e}(\mathbf{q}, \mathbf{p})=\boldsymbol{\epsilon}$, are related through two equations:

$$
\begin{aligned}
\langle\mathbf{l}, \boldsymbol{\epsilon}\rangle & =0, \\
\epsilon^{2}-1 & =\frac{2 \mathbf{l}^{2}}{\mu^{2}} h,
\end{aligned}
$$

\footnotetext{
${ }^{1}$ We shall use this standard notation henceforth: for every $\mathbf{x} \in \mathbb{R}^{n}$ we set $x=|\mathbf{x}|$.
} 
which defines a smooth, five dimensional sub-manifold $\mathcal{M}$ in $(\mathbf{l}, \boldsymbol{\epsilon}, h)$-space. In other words, there exists five independent conserved quantities.

It follows from (2) and (3) that the eccentricity vector is in the orbital plane and its magnitude can be determined from $\mathbf{L}$ and $H$, hence only the direction of the eccentricity vector is conserved independently. Moreover, by taking the dot product of $\mathbf{e}$ with $\mathbf{q}$ one finds that $t \mapsto q(t)$ traces out a conic section determined by the norm of the eccentricity: elliptic $\epsilon<1$, parabolic $\epsilon=1$ or hyperbolic if $\epsilon>1$.

For $\epsilon<1$ a qualitative description of the relative motion of satellites on Keplerian orbits can be obtained from Kepler's third law. This says that the period of the periodic orbit only depends upon the semi-major axis $a$, and since the energy constant, $h$, is directly related to the semi-major axis:

$$
h=-\frac{\mu}{2 a},
$$

it follows that neighbouring satellites with different energies will evidently drift apart. For $\epsilon<1$ variations in the other constants of motion only give rise to bounded relative motion. In the following section we introduce a general Hamiltonian setting to variational equations.

\section{General Hamiltonian setting of variational equations}

For any Hamiltonian $H$ on a symplectic manifold $\left(M=T^{*} Q, \omega\right)$, Hamilton's equations in local coordinates:

$$
\begin{aligned}
& \dot{\mathbf{q}}=\partial_{\mathbf{p}} H(\mathbf{q}, \mathbf{p}), \\
& \dot{\mathbf{p}}=-\partial_{\mathbf{q}} H(\mathbf{q}, \mathbf{p}),
\end{aligned}
$$

can be written more compactly as

$$
\dot{\mathbf{u}}=\mathbf{J} \nabla H(\mathbf{u})
$$

where $\mathbf{u}=(\mathbf{q}, \mathbf{p})$ and $\mathbf{J}$ is the Poisson-matrix

$$
\mathbf{J}=\left(\begin{array}{cc}
\mathbf{0} & \mathbf{I} \\
-\mathbf{I} & \mathbf{0}
\end{array}\right)
$$


with $\mathbf{I}$ identity in $\mathbb{R}^{n \times n}$. The variational equations may then be written as

$$
\dot{\boldsymbol{\delta} \mathbf{u}}=\mathbf{J} \nabla^{2} H(\mathbf{u}) \boldsymbol{\delta} \mathbf{u}
$$

where $\nabla^{2} H$ is the Hessian.

Hamilton's equations may also be written in a coordinate independent way from the symplectic form: the Hamiltonian vector field is the unique vector field $\mathbf{X}_{h}$ satisfying

$$
\omega\left(\mathbf{X}_{h}(\mathbf{u}), \mathbf{w}\right)=\delta H(\mathbf{u})(\mathbf{w}), \quad \forall \mathbf{w} \in T_{\mathbf{u}} M
$$

By variations we may define the variational vector field $\mathbf{X}_{\delta H}: T M \rightarrow T(T M)$ as the unique vector field satisfying

$$
\delta \omega\left(\mathbf{X}_{\delta H}(\mathbf{u}, \boldsymbol{\delta} \mathbf{u}),(\mathbf{w}, \boldsymbol{\delta} \mathbf{w})\right)=\delta(\delta H(\mathbf{u})(\boldsymbol{\delta} \mathbf{u}))(\mathbf{w}, \boldsymbol{\delta} \mathbf{w}), \quad \forall(\mathbf{w}, \boldsymbol{\delta} \mathbf{w}) \in T_{(\mathbf{u}, \delta \mathbf{u})}(T M)
$$

Here $\delta \omega$ is a sympletic form on $T M$, see e.g. [14], and (7) is the coordinate independent version of (5) and (6).

Let $G$ be a Lie-group acting smoothly upon $M=T^{*} Q$ that leaves $H$ invariant:

$$
H(g \cdot \mathbf{u})=H(\mathbf{u}), \quad \forall g \in G, \forall \mathbf{u} \in M
$$

By Noether's theorem there exist an associated conserved quantity $C=C(\mathbf{u})$ of dimension equal to the dimension of $G$. The $G$-action lifts naturally to an action on $T M$, and from (8) it directly follows that

$$
\delta H(g \cdot \mathbf{u})(\delta g \cdot \boldsymbol{\delta} \mathbf{u})=\delta H(\mathbf{u})(\boldsymbol{\delta} \mathbf{u})
$$

and hence any $G$-invariance of $H$ translates to $G$-invariance of $\delta H$. As a corollary any $(G$ equivariant) conserved quantity, $C(\mathbf{u})$, of the original system give rise to a (G-equivariant) conserved quantity, $\delta C$, of the Hamiltonian system $\left(T^{*} M, \delta H, \delta \omega\right)$. In coordinates:

$$
\delta C(\mathbf{u})(\boldsymbol{\delta} \mathbf{u})=\langle\nabla C(\mathbf{u}), \boldsymbol{\delta} \mathbf{u}\rangle
$$

For the particular case of

$$
H(\mathbf{q}, \mathbf{p})=\frac{1}{2}|\mathbf{p}|^{2}-\frac{\mu}{|\mathbf{q}|}
$$


the variational Hamiltonian is

$$
\delta H(\mathbf{q}, \mathbf{p}, \boldsymbol{\delta} \mathbf{q}, \boldsymbol{\delta} \mathbf{p})=\langle\mathbf{p}, \boldsymbol{\delta} \mathbf{p}\rangle+\mu \frac{\mathbf{q}}{|\mathbf{q}|^{3}}\langle\mathbf{q}, \delta \mathbf{q}\rangle
$$

with $\delta \omega=d \boldsymbol{\delta} \mathbf{q} \wedge d \mathbf{p}+d \mathbf{q} \wedge d \boldsymbol{\delta} \mathbf{p}$ and

$$
X_{\delta H}=\left(\begin{array}{c}
\mathbf{p} \\
-\frac{\mu}{q^{3}} \mathbf{q} \\
\delta \mathbf{p} \\
-\frac{\mu}{q^{3}}\left(I-3 \frac{\mathbf{q q}}{q^{2}}\right) \delta \mathbf{q}
\end{array}\right) .
$$

Moreover, by (10)

$$
\begin{aligned}
\delta \mathbf{L}(\mathbf{q}, \mathbf{p}, \delta \mathbf{q}, \delta \mathbf{p}) & =\delta \mathbf{q} \wedge \mathbf{p}+\mathbf{q} \wedge \delta \mathbf{p} \\
\delta \mathbf{e}(\mathbf{q}, \mathbf{p}, \delta \mathbf{q}, \delta \mathbf{p}) & =-\frac{\delta \mathbf{q}}{|\mathbf{q}|}+\frac{\langle\mathbf{q}, \delta \mathbf{q}\rangle \mathbf{q}}{|\mathbf{q}|^{3}}+\frac{1}{\mu} \delta \mathbf{p} \wedge \mathbf{L}(\mathbf{q}, \mathbf{p}) \\
& +\frac{1}{\mu} \mathbf{p} \wedge \delta \mathbf{L}(\mathbf{q}, \mathbf{p}, \delta \mathbf{q}, \delta \mathbf{p})
\end{aligned}
$$

are also conserved. We call the quantities (11) and (12) and (13) the 'relative Hamiltonian', 'the relative angular momentum' and 'relative eccentricity', respectively, as in Palmer and Imre [16]. The variables $(\boldsymbol{\delta} \mathbf{q}, \boldsymbol{\delta} \mathbf{p})$ are first order approximations to the relative motion of two neighbouring satellites near their centre of mass orbit at (q, p).

Setting $\delta H(\mathbf{u}, \boldsymbol{\delta} \mathbf{u})=\delta h, \boldsymbol{\delta} \mathbf{L}(\mathbf{u}, \boldsymbol{\delta} \mathbf{u})=\boldsymbol{\delta} \mathbf{l}$ and $\boldsymbol{\delta} \mathbf{e}(\mathbf{u}, \boldsymbol{\delta} \mathbf{u})=\boldsymbol{\delta} \boldsymbol{\epsilon}$ from (2) and (3) we obtain the following relations:

$$
\begin{aligned}
\langle\boldsymbol{\delta} \mathbf{l}, \mathbf{e}\rangle+\langle\mathbf{l}, \boldsymbol{\delta} \boldsymbol{\epsilon}\rangle & =0 \\
2\langle\boldsymbol{\epsilon}, \boldsymbol{\delta} \boldsymbol{\epsilon}\rangle & =\frac{4\langle\mathbf{l}, \boldsymbol{\delta} \mathbf{l}\rangle}{\mu^{2}} h+\frac{2 l^{2}}{\mu^{2}} \delta h .
\end{aligned}
$$

The main tool used in our construction of the variational solutions will rely on the following. Since $2 n-1$ ( $n=$ the degrees of freedom) independent quantities are conserved, the Kepler problem falls into the category of maximally super-integrable systems [21], and we may express its solutions in terms of $h, \mathbf{l}, \boldsymbol{\epsilon}$, and time in the form $t-t_{0}$, i.e.

$$
\mathbf{u}:=(\mathbf{q}, \mathbf{p})=\mathbf{u}\left(h, \mathbf{l}, \boldsymbol{\epsilon}, t-t_{0}\right)
$$


As a consequence of the chain rule and the rule of mixed partials, solutions of the variational

equations can be obtained by taking independent variations with respect to $h, \mathbf{l}, \boldsymbol{\epsilon}$ and $t_{0}$, see also Wiesel and Pohlen [24]. Indeed, let $\mathbf{c} \in \mathbb{R}^{5}$ be coordinates on the five dimensional sub-manifold, $\mathcal{M}$, embedded within $(h, \mathbf{l}, \boldsymbol{\epsilon})$-space and by the identities $(2)$ and $(3)$, and view $\mathbf{u}\left(t-t_{0}\right)$ as a vector-valued function on $\mathcal{M}$. Then

$$
\boldsymbol{\delta} \mathbf{u}\left(t-t_{0}\right)(\mathbf{c})(\boldsymbol{\delta} \mathbf{c})=\sum_{i=1}^{5} \partial_{c_{i}} \mathbf{u}\left(t-t_{0}\right)(\mathbf{c}) \delta c_{i}, \quad \boldsymbol{\delta} \mathbf{c} \in T_{\mathbf{c}} \mathcal{M} \cong \mathbb{R}^{5}
$$

Differentiation with respect to $t$ gives

$$
\begin{aligned}
d_{t}\left[\partial_{c_{i}} \mathbf{u}\left(t-t_{0}\right)(\mathbf{c}) \delta c_{i}\right] & =\partial_{c_{i}} \dot{\mathbf{u}}\left(t-t_{0}\right) \delta c_{i} \\
& =\partial_{c_{i}} \mathbf{J} \nabla_{\mathbf{u}} H\left(\mathbf{u}\left(t-t_{0}\right)(\mathbf{c}) \delta c_{i}\right. \\
& =\mathbf{J} \nabla_{\mathbf{u}}^{2} H\left(\mathbf{u}\left(t-t_{0}\right)(\mathbf{c})\right)\left[\partial_{c_{i}} \mathbf{u}\left(t-t_{0}\right)(\mathbf{c}) \delta c_{i}\right],
\end{aligned}
$$

showing that $\partial_{c_{i}} \mathbf{u}\left(t-t_{0}\right)(\mathbf{c}) \delta c_{i}$ for $i=1, \ldots, 5$ solve the variational equations. Fixing $\mathbf{c}$ and instead viewing $\mathbf{u}(t)$ as a vector-valued function of $t_{0}$, which enters in the form $t-t_{0}$, we obtain that

$$
\boldsymbol{\delta} \mathbf{u}(t)\left(t_{0}\right)\left(\delta t_{0}\right)=\dot{\mathbf{u}} \delta\left(-t_{0}\right)=-\dot{\mathbf{u}} \delta t_{0}
$$

i.e. the vector field along the reference orbit, is a solution of the variational problem. This is a well-known fact. In the following section we aim to determine the solution of the variational equations of Kepler's problem by exploiting this geometrical setting.

\section{Solutions of Kepler's variational equations}

If, for $\epsilon \neq 0,1$,

$$
\begin{aligned}
& \boldsymbol{\delta} \mathbf{L}(\mathbf{u}, \boldsymbol{\delta} \mathbf{u})=\boldsymbol{\delta} \mathbf{l}=\delta l_{1} \mathbf{i}+\delta l_{2} \mathbf{j}+\delta l_{3} \mathbf{k} \\
& \boldsymbol{\delta} \mathbf{e}(\mathbf{u}, \boldsymbol{\delta} \mathbf{u})=\boldsymbol{\delta} \boldsymbol{\epsilon}=\delta \epsilon_{1} \mathbf{i}+\delta \epsilon_{2} \mathbf{j}+\delta \epsilon_{3} \mathbf{k}
\end{aligned}
$$


where $\mathbf{i}=\frac{\boldsymbol{\epsilon}}{\epsilon}, \mathbf{j}=\frac{\mathbf{l} \wedge \boldsymbol{\epsilon}}{l \wedge \epsilon}$ and $\mathbf{k}=\frac{1}{l}$, see Fig. 1, then we may write (14) and (15) in coordinates as:

$$
\begin{aligned}
\delta \epsilon_{3} & =-\frac{\epsilon}{l} \delta l_{1}, \\
\frac{\epsilon}{1-\epsilon^{2}} \delta \epsilon_{1}+\frac{\delta l_{3}}{l} & =-\frac{\delta h}{2 h} .
\end{aligned}
$$

We shall return to $\epsilon=0,1$ later.

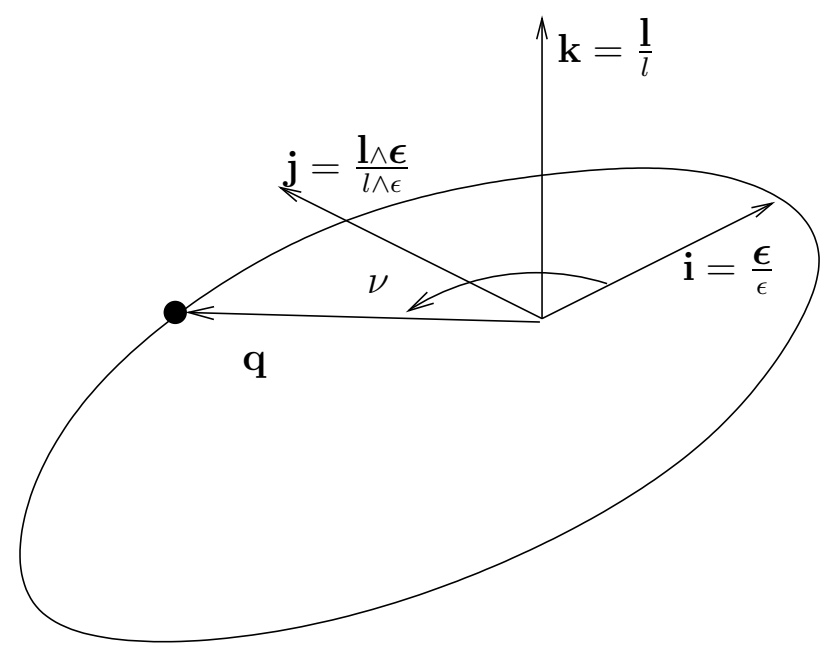

Figure 1: Orthogonal basis $(\mathbf{i}, \mathbf{j}, \mathbf{k})$.

For $\epsilon \neq 1$ (17) and (18) show that we may describe every variation using $\boldsymbol{\delta} \mathbf{l}, \delta \epsilon_{1}$ and $\delta \epsilon_{2}$. Writing

$$
\mathbf{q}(t ; \mathbf{l}, \boldsymbol{\epsilon})=q(\mathbf{i} \cos \nu+\mathbf{j} \sin \nu),
$$

where everything on the right hand side is a function of $t-t_{0}, \mathbf{l}$ and $\boldsymbol{\epsilon}$, we obtain

$$
\begin{aligned}
\boldsymbol{\delta} \mathbf{q} & =\delta q_{1} \mathbf{i}+\delta q_{2} \mathbf{j}+q_{1} \underbrace{\left(\frac{\boldsymbol{\delta} \boldsymbol{\epsilon}}{\epsilon}-\frac{\boldsymbol{\epsilon}}{\epsilon^{3}}\langle\boldsymbol{\epsilon}, \boldsymbol{\delta} \boldsymbol{\epsilon}\rangle\right)}_{\boldsymbol{\delta}_{\mathbf{i}}} \\
& +q_{2} \underbrace{\left(\frac{\boldsymbol{\delta} \mathbf{l} \wedge \boldsymbol{\epsilon}+\mathbf{l} \wedge \boldsymbol{\delta} \boldsymbol{\epsilon}}{l \wedge \epsilon}-\frac{1 \wedge \boldsymbol{\epsilon}}{(l \wedge \epsilon)^{3}}\langle\mathbf{l} \wedge \boldsymbol{\epsilon}, \boldsymbol{\delta} \mathbf{l} \wedge \boldsymbol{\epsilon}+\mathbf{l} \wedge \boldsymbol{\delta} \boldsymbol{\epsilon}\rangle\right)}_{\boldsymbol{\delta}_{\mathbf{j}}}, \quad \epsilon \neq 0,1,
\end{aligned}
$$

where $q_{1}:=q \cos \nu$ and $q_{2}:=q \sin \nu$ with $q=l^{2} /(\mu(1+\epsilon \cos \nu))$. 
Note that $q_{1}$ and $q_{2}$ only depend upon the norm of $\boldsymbol{\epsilon}$ and $\mathbf{l}$. Since $\epsilon=\epsilon_{1}$ and $l=l_{3}$ we obtain:

$$
\begin{aligned}
& \delta q_{1}=\partial_{l} q_{1} \delta l_{3}+\partial_{\epsilon} q_{1} \delta \epsilon_{1}, \\
& \delta q_{2}=\partial_{l} q_{2} \delta l_{3}+\partial_{\epsilon} q_{2} \delta \epsilon_{1} .
\end{aligned}
$$

By linearity we can consider the variations independently. Therefore, first let $\delta l_{3}=0$ and $\delta \epsilon_{1}=0$. Then (19) gives

$$
\boldsymbol{\delta} \mathbf{q}=-\frac{q_{2}}{\epsilon} \delta \epsilon_{1} \mathbf{i}+\frac{q_{1}}{\epsilon} \delta \epsilon_{2} \mathbf{j}-\left(q_{1} \delta l_{1}+q_{2} \frac{\epsilon}{l(l \wedge \epsilon)} \delta l_{2}\right) \mathbf{k}, \quad \epsilon \neq 0,1
$$

or compactly:

$$
\boldsymbol{\delta} \mathbf{q}=\frac{\mathbf{l} \wedge \mathbf{q}}{l \wedge \epsilon} \delta \epsilon_{2}+\frac{(\mathbf{l} \wedge \boldsymbol{\epsilon}) \wedge \mathbf{q}}{l(l \wedge \epsilon)} \delta l_{1}+\frac{\boldsymbol{\epsilon} \wedge \mathbf{q}}{l \wedge \epsilon} \delta l_{2}, \quad \epsilon \neq 0,1 .
$$

Consider $\delta l_{1}=0=\delta l_{2}$ and $\delta \epsilon_{2}=0$, so that $\boldsymbol{\delta} \mathbf{i}=\mathbf{0}=\boldsymbol{\delta} \mathbf{j}$. Then (19) becomes:

$$
\begin{aligned}
\boldsymbol{\delta} \mathbf{q} & =\delta q_{1} \mathbf{i}+\delta q_{2} \mathbf{j} \\
& =\left(\partial_{l} q_{1} \mathbf{i}+\partial_{l} q_{2} \mathbf{j}\right) \delta l_{3}+\left(\partial_{\epsilon} q_{1} \mathbf{i}+\partial_{\epsilon} q_{2} \mathbf{j}\right) \delta \epsilon_{1}
\end{aligned}
$$

The partial derivatives of $q_{1}$ and $q_{2}$ are:

$$
\begin{aligned}
& \partial_{l} q_{1}=\underbrace{\frac{2 l / \mu}{1+\epsilon \cos \nu} \cos \nu}_{2 q_{1} / l}+\underbrace{\frac{l^{2}}{\mu} \frac{-\sin \nu}{(1+\epsilon \cos \nu)^{2}}}_{=\partial_{\nu} q_{1}} \partial_{l} \nu, \\
& \partial_{l} q_{2}=\underbrace{\frac{2 l / \mu}{1+\epsilon \cos \nu} \sin \nu}_{2 q_{2} / l}+\underbrace{\frac{l^{2}}{\mu} \frac{\epsilon+\cos \nu}{(1+\epsilon \cos \nu)^{2}}}_{=\partial_{\nu} q_{2}} \partial_{l} \nu, \\
& \partial_{\epsilon} q_{1}=-\frac{l^{2} / \mu}{(1+\epsilon \cos \nu)^{2}} \cos ^{2} \nu+\frac{l^{2}}{\mu} \frac{-\sin \nu}{(1+\epsilon \cos \nu)^{2}} \partial_{\epsilon} \nu, \\
& \partial_{\epsilon} q_{2}=-\frac{l^{2} / \mu}{(1+\epsilon \cos \nu)^{2}} \cos \nu \sin \nu+\frac{l^{2}}{\mu} \frac{\epsilon+\cos \nu}{(1+\epsilon \cos \nu)^{2}} \partial_{\epsilon} \nu .
\end{aligned}
$$

To obtain the partial derivatives of $\nu$ we separate variables of the differential equation of $\nu=\nu(t):$

$$
\dot{\nu}=\frac{\mu^{2}}{l^{3}}(1+\epsilon \cos \nu)^{2}
$$


which follows from conservation of angular momentum: $q^{2} \dot{\nu}=l$, to obtain

$$
\frac{2}{\left(1-\epsilon^{2}\right)^{3 / 2}} \arctan \left(\frac{(1-\epsilon)(1-\cos \nu)}{\sqrt{1-\epsilon^{2}} \sin \nu}\right)=\frac{\mu^{2}}{l^{3}}\left(t-t_{0}\right)+\frac{\epsilon}{\left(1-\epsilon^{2}\right)(1+\epsilon \cos \nu)} \sin \nu .
$$

This equality extends to $\epsilon>1$ upon replacing arctan with arctanh. Differentiation of (25) with respect to $l$ gives:

$$
\partial_{l} \nu=-\frac{3}{l} \underbrace{\frac{\mu^{2}}{l^{3}}(1+\epsilon \cos \nu)^{2}}_{=d_{t} \nu}\left(t-t_{0}\right)
$$

which, by continuity of $\nu$ as a function of $\epsilon$ and $l$, extends to $\epsilon=1, l \neq 0$. Next, for $\epsilon \neq 1$ differentiation with respect to $\epsilon$ gives, after some manipulations:

$$
\partial_{\epsilon} \nu=-\frac{3 \epsilon}{1-\epsilon^{2}} \frac{\mu^{2}}{l^{3}}(1+\epsilon \cos \nu)^{2}\left(t-t_{0}\right)+\frac{\sin \nu(2+\epsilon \cos \nu)}{1-\epsilon^{2}}, \quad \epsilon \neq 1
$$

Notice the singularities for $\epsilon=1$ and $l=0$. We will return to these cases in section 4.2. Using (21) and (22) we arrive at:

$$
\begin{aligned}
\left(\partial_{l} q_{1} \mathbf{i}+\partial_{l} q_{2} \mathbf{j}\right) \delta l_{3} & =2\left(\mathbf{q}-\frac{3}{2} \mathbf{p}\left(t-t_{0}\right)\right) \frac{\delta l_{3}}{l} \\
\left(\partial_{\epsilon} q_{1} \mathbf{i}+\partial_{\epsilon} q_{2} \mathbf{j}\right) \delta \epsilon_{1} & =-\frac{3 \epsilon}{1-\epsilon^{2}} \mathbf{p}\left(t-t_{0}\right) \delta \epsilon_{1} \\
& +\frac{l^{2} / \mu}{(1+\epsilon \cos \nu)^{2}}\left(\epsilon^{2} \cos ^{2} \nu-1-\sin ^{2} \nu(1+\epsilon \cos \nu)\right) \mathbf{i} \frac{\delta \epsilon_{1}}{1-\epsilon^{2}} \\
& +\frac{l^{2} / \mu}{(1+\epsilon \cos \nu)^{2}} \sin \nu\left((\epsilon+\cos \nu)(2+\epsilon \cos \nu)-\cos \nu\left(1-\epsilon^{2}\right)\right) \mathbf{j} \frac{\delta \epsilon_{1}}{1-\epsilon^{2}}
\end{aligned}
$$

By (18):

$$
\begin{array}{r}
-3 \mathbf{p}\left(t-t_{0}\right) \frac{\delta l_{3}}{l}-\frac{3 \epsilon}{1-\epsilon^{2}} \mathbf{p}\left(t-t_{0}\right) \delta \epsilon_{1}=\frac{3}{2} \mathbf{p}\left(t-t_{0}\right) \frac{-\delta h}{h} \\
2 q \frac{\delta l_{1}}{l}=q \frac{-\delta h}{h}-2 \mathbf{q} \frac{\epsilon \delta \epsilon_{1}}{1-\epsilon^{2}}
\end{array}
$$


so that, in terms of $\delta h$ and $\delta \epsilon_{1}$, we finally obtain:

$$
\begin{aligned}
& \left(\partial_{l} q_{1} \mathbf{i}+\partial_{l} q_{2} \mathbf{j}\right) \delta l_{3}+\left(\partial_{\epsilon} q_{1} \mathbf{i}+\partial_{\epsilon} q_{2} \mathbf{j}\right) \delta \epsilon_{1} \\
& =\left(\mathbf{q}-\frac{3}{2} \mathbf{p}\left(t-t_{0}\right)\right) \frac{-\delta h}{h}-2 \frac{\epsilon}{1-\epsilon^{2}} \mathbf{q} \delta \epsilon_{1} \\
& +\frac{l^{2} / \mu}{(1+\epsilon \cos \nu)^{2}}\left(\epsilon^{2} \cos ^{2} \nu-1-\sin ^{2} \nu(1+\epsilon \cos \nu)\right) \mathbf{i} \frac{\delta \epsilon_{1}}{1-\epsilon^{2}} \\
& +\frac{l^{2} / \mu}{(1+\epsilon \cos \nu)^{2}} \sin \nu\left((\epsilon+\cos \nu)(2+\epsilon \cos \nu)-\cos \nu\left(1-\epsilon^{2}\right)\right) \mathbf{j} \frac{\delta \epsilon_{1}}{1-\epsilon^{2}} \\
& =\left(\mathbf{q}-\frac{3}{2} \mathbf{p}\left(t-t_{0}\right)\right) \frac{-\delta h}{h} \\
& -\frac{l^{2}}{\mu}\left(1+\frac{\sin ^{2} \nu}{1+\epsilon \cos \nu}\right) \mathbf{i} \frac{\delta \epsilon_{1}}{1-\epsilon^{2}} \\
& +\frac{l^{2} / \mu}{1+\epsilon \cos \nu} \sin \nu \cos \nu \mathbf{j} \frac{\delta \epsilon_{1}}{1-\epsilon^{2}}
\end{aligned}
$$

or compactly:

$$
\begin{aligned}
& \left(\partial_{l} q_{1} \mathbf{i}+\partial_{l} q_{2} \mathbf{j}\right) \delta l_{3}+\left(\partial_{\epsilon} q_{1} \mathbf{i}+\partial_{\epsilon} q_{2} \mathbf{j}\right) \delta \epsilon_{1} \\
& =\left(\mathbf{q}-\frac{3}{2} \mathbf{p}\left(t-t_{0}\right)\right) \frac{-\delta h}{h}-\left(\frac{l^{2}}{\mu} \mathbf{i}+\sin \nu \mathbf{q} \wedge \mathbf{k}\right) \frac{1}{1-\epsilon^{2}} \delta \epsilon_{1} .
\end{aligned}
$$

For $\epsilon \neq 0,1$ we have therefore constructed the variational solution:

$$
\begin{aligned}
\boldsymbol{\delta} \mathbf{q}\left(t ; \delta t_{0}, \boldsymbol{\delta} \boldsymbol{\epsilon}, \boldsymbol{\delta} \mathbf{l}, \delta h\right) & =-\mathbf{p} \delta t_{0}+\mathbf{k} \wedge \mathbf{q} \frac{\delta \epsilon_{2}}{\epsilon}+\mathbf{j} \wedge \mathbf{q} \frac{\delta l_{1}}{l}+\mathbf{i} \wedge \mathbf{q} \frac{\delta l_{2}}{l} \\
& +\left(\mathbf{q}-\frac{3}{2} \mathbf{p}\left(t-t_{0}\right)\right) \frac{-\delta h}{h}-\left(\frac{l^{2}}{\mu} \mathbf{i}+\sin \nu \mathbf{q} \wedge \mathbf{k}\right) \frac{1}{1-\epsilon^{2}} \delta \epsilon_{1}
\end{aligned}
$$

\subsection{Circular reference orbits: $\epsilon=0$}

When $\epsilon=0$ then $\mathbf{i}$ and $\mathbf{j}$ are not well-defined as the circular motion is completely isotropic, and hence variations $\boldsymbol{\delta} \mathbf{i}$ and $\boldsymbol{\delta} \mathbf{j}$ are singular in $\epsilon$. As a consequence, one may note that for $\epsilon=0, \mathbf{l} \wedge \mathbf{q}=\frac{l^{4}}{\mu^{2}} \mathbf{p}$ and the solution obtained by differentiating the vector field along the reference orbit, (16), is therefore proportional to the solution corresponding to variations in $\epsilon_{2}$ which appears as the second term on the right hand side of (30). However, $\mathbf{k}$ is still well-defined and defines the orbital plane in which we may choose any orthonormal basis $\{\boldsymbol{\iota}, \boldsymbol{\lambda}\} \subset P(1)$. To account for variations in $\boldsymbol{\epsilon}$ at $\mathbf{0}$ we may take two independent variations 
e.g. along the direction of $\iota$ and $\boldsymbol{\lambda}$. In detail, we consider two eccentric orbits with eccentricity vector pointing in the direction of $\iota$ and $\boldsymbol{\lambda}$ :

$$
\begin{aligned}
& \mathbf{q}=\frac{l^{2} / \mu}{1+\epsilon \cos \nu}(\cos \nu \iota+\sin \nu \boldsymbol{\lambda}), \\
& \text { respectively } \\
& \mathbf{q}=\frac{l^{2} / \mu}{1+\epsilon \sin \nu}(\cos \nu \iota+\sin \nu \boldsymbol{\lambda}),
\end{aligned}
$$

see also Fig. 2, and take linear variations with respect to $\epsilon$ at $\epsilon=0$ to obtain:

$$
\begin{aligned}
\delta \mathbf{q} & =l^{2} / \mu\left(-\left(1+\sin ^{2} \nu\right) \delta \epsilon_{1}+\cos \nu \sin \nu \delta \epsilon_{2}\right) \boldsymbol{\iota} \\
& +l^{2} / \mu\left(\cos \nu \sin \nu \delta \epsilon_{1}-\left(1+\cos ^{2} \nu\right) \delta \epsilon_{2}\right) \boldsymbol{\lambda} .
\end{aligned}
$$

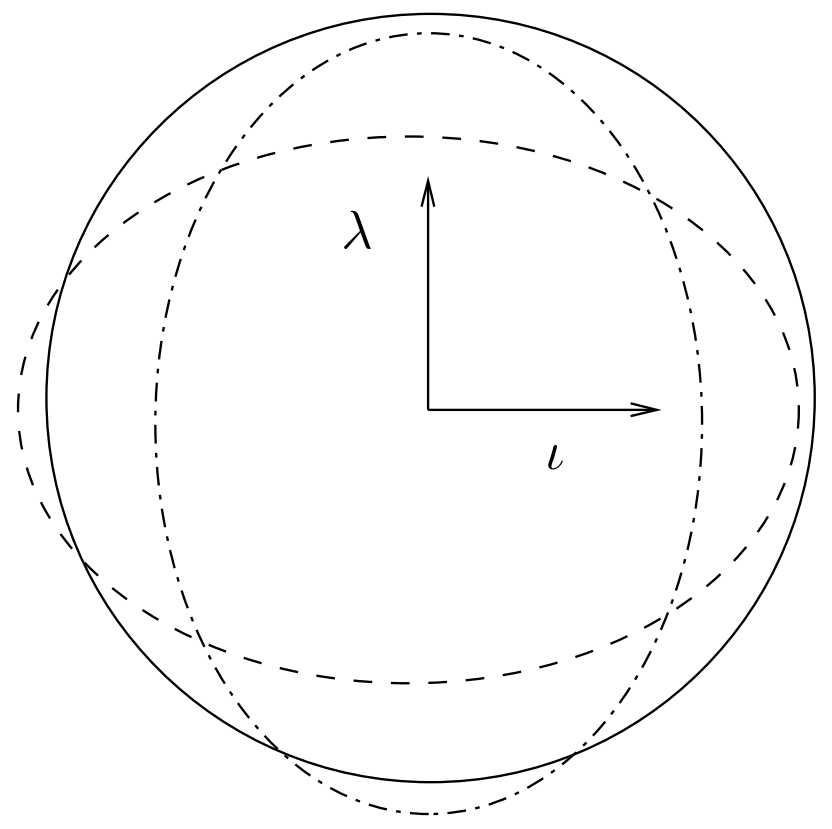

Figure 2: Two elliptic sections, whose eccentricity vectors are mutually orthogonal in the orbital plane, near a circular orbit.

The terms in the complete solution (30) describing the variation of $\delta t_{0}, \delta l_{1}, \delta l_{2}$ and $\delta h$ 
are still valid for $\epsilon=0$ when replacing $\mathbf{i}$ and $\mathbf{j}$ with $\boldsymbol{\iota}$ and $\boldsymbol{\lambda}$ respectively. Therefore for $\epsilon=0$ :

$$
\begin{aligned}
\boldsymbol{\delta} \mathbf{q}\left(t ; \delta t_{0}, \boldsymbol{\delta} \boldsymbol{\epsilon}, \boldsymbol{\delta} \mathbf{l}, \delta h\right) & =-\mathbf{p} \delta t_{0}+\frac{\boldsymbol{\lambda} \wedge \mathbf{q}}{l} \delta l_{1}+\frac{\boldsymbol{\iota} \wedge \mathbf{q}}{l} \delta l_{2}+\left(\mathbf{q}-\frac{3}{2} \mathbf{p}\left(t-t_{0}\right)\right) \frac{-\delta h}{h} \\
& +l^{2} / \mu\left(-\left(1+\sin ^{2} \nu\right) \delta \epsilon_{1}+\cos \nu \sin \nu \delta \epsilon_{2}\right) \iota \\
& +l^{2} / \mu\left(\cos \nu \sin \nu \delta \epsilon_{1}-\left(1+\cos ^{2} \nu\right) \delta \epsilon_{2}\right) \boldsymbol{\lambda} .
\end{aligned}
$$

By moving into a rotating frame it is straightforward to show that the solutions coincide with the solutions of the Hill-Clohessy-Wiltshire equations.

\subsection{Parabolic reference orbits: $\epsilon=1$}

When $\epsilon=1$ then by (3) either $l=0$ or $h=0$. If $l=0$ then the motion is purely radial, $\dot{\nu}=0$. Let us initially assume that $l \neq 0$ and therefore $h=0$. The solution in (30) does not extend trivially to $\epsilon=1$ due to the singularity in $\partial_{\epsilon} \nu(27)$. However, the singularity is removable by continuity of $\nu$ as a function of $l$ and $\epsilon$. By successive application of L'Hôpital's rule to (25), we arrive at:

$$
\left.\partial_{\epsilon} \nu\right|_{\epsilon=1}=-\frac{2}{5} \frac{\sin \nu\left(1+3 \cos \nu+\cos ^{2} \nu\right)}{1+\cos \nu}
$$

Inserting this into (23) and (24) we then obtain the variations in $\mathbf{q}$ due to variations in $\epsilon_{1}$ :

$$
\begin{aligned}
\left.\left(\partial_{\epsilon} q_{1} \mathbf{i}+\partial_{\epsilon} q_{2} \mathbf{j}\right)\right|_{\epsilon=1} \delta \epsilon_{1} & =\frac{l^{2} / \mu}{5(1+\epsilon \cos \nu)^{2}}\left(2+4 \cos \nu-9 \cos ^{2} \nu-2 \cos ^{3} \nu\right) \mathbf{i} \delta \epsilon_{1} \\
& -\frac{l^{2} / \mu}{5(1+\epsilon \cos \nu)^{2}} \sin \nu\left(2+2 \cos \nu+2 \cos ^{2} \nu\right) \mathbf{j} \delta \epsilon_{1} .
\end{aligned}
$$

On the other hand, $\partial_{l} \nu(26)$ extends trivially:

$$
\left.\partial_{l} \nu\right|_{\epsilon=1}=-\frac{3}{l} \frac{\mu^{2}}{l^{3}}(1+\cos \nu)^{2}\left(t-t_{0}\right)
$$

and so does the variations with respect $l_{3}(28)$ :

$$
\left.\left(\partial_{l} q_{1} \mathbf{i}+\partial_{l} q_{2} \mathbf{j}\right)\right|_{\epsilon=1} \delta l_{3}=2\left(\mathbf{q}-\frac{3}{2} \mathbf{p}\left(t-t_{0}\right)\right) \frac{\delta l_{3}}{l} .
$$


For $\epsilon=1, h=0, l \neq 0$ we have therefore constructed the following:

$$
\begin{aligned}
\delta \mathbf{q}\left(t ; \delta t_{0}, \boldsymbol{\delta} \boldsymbol{\epsilon}, \boldsymbol{\delta} \mathbf{l}, \delta h\right) & =-\mathbf{p} \delta t_{0}+\mathbf{k} \wedge \mathbf{q} \delta \epsilon_{2}+\mathbf{j} \wedge \mathbf{q} \frac{\delta l_{1}}{l}+\mathbf{i} \wedge \mathbf{q} \frac{\delta l_{2}}{l} \\
& +2\left(\mathbf{q}-\frac{3}{2} \mathbf{p}\left(t-t_{0}\right)\right) \frac{\delta l_{3}}{l} \\
& +\frac{2 l^{4} / \mu^{3}}{5(1+\cos \nu)^{2}}\left(2+4 \cos \nu-9 \cos ^{2} \nu-2 \cos ^{3} \nu\right) \mathbf{i} \delta h \\
& -\frac{2 l^{4} / \mu^{3}}{5(1+\cos \nu)^{2}} \sin \nu\left(11 \cos \nu+2\left(1+\cos ^{2} \nu\right)\right) \mathbf{j} \delta h,
\end{aligned}
$$

where we have used $\delta \epsilon_{1}=\frac{2 l^{2}}{\mu^{2}} \delta h$ for $\epsilon=1$ and $h=0$.

Next, we let $l=0$ and $h \neq 0$. Then $\mathbf{q}$ is purely radial, having only a component in the direction of the eccentricity vector, say $\mathbf{q}=q_{1} \boldsymbol{\epsilon}=q_{1} \mathbf{i}, q_{1}>0$, and may, due to the possibility of collision with the central body only exist for finite time. Letting $(\mathbf{j}, \mathbf{k})$ be an orthonormal basis for the plane perpendicular to the $\mathbf{i}=\boldsymbol{\epsilon}$ direction it again can be seen that $\mathbf{j} \wedge \mathbf{q}$ and $\mathbf{k} \wedge \mathbf{q}$ are independent solutions corresponding to variations in $\delta \epsilon_{2}$ and $\delta \epsilon_{3}$ respectively. By insertion one may also verify that $\left(\mathbf{q}-\frac{3}{2} \mathbf{p}\left(t-t_{0}\right)\right) \frac{-\delta h}{h}$ is a solution. Therefore, for $l=0$ and $h \neq 0$ we have so far four linearly independent solutions of the variational problem:

$$
\boldsymbol{\delta} \mathbf{q}=-\mathbf{p} \delta t_{0}-\mathbf{k} \wedge \mathbf{q} \delta \epsilon_{2}+\mathbf{j} \wedge \mathbf{q} \delta \epsilon_{3}+\left(\mathbf{q}-\frac{3}{2} \mathbf{p}\left(t-t_{0}\right)\right) \frac{-\delta h}{h} .
$$

To obtain the full solution of the linear problem:

$$
\begin{aligned}
& \dot{\boldsymbol{\delta} \mathbf{q}}=\boldsymbol{\delta} \mathbf{p} \\
& \dot{\delta \mathbf{p}}=\frac{\mu}{q_{1}^{3}} \operatorname{diag}(2,-1,-1) \delta \mathbf{q},
\end{aligned}
$$

only two additional, linearly independent solutions are required. For these let us assume $\boldsymbol{\delta} \mathbf{q}=\left(0, \delta q_{2}, 0\right), \boldsymbol{\delta} \mathbf{p}=\left(0, \delta p_{2}, 0\right)$. Then $\boldsymbol{\delta} \mathbf{L}$ explicitly reads:

$$
\boldsymbol{\delta} \mathbf{L}=\left(0,0, q_{1} \delta p_{2}-p_{1} \delta q_{2}\right)
$$

From the conservation of $\boldsymbol{\delta} \mathbf{L}=\left(0,0, \delta l_{3}\right)$ we obtain, using $\delta p_{2}=\dot{\delta} q_{2}$, a first order, ordinary differential equation for $\delta q_{2}$ :

$$
\begin{aligned}
\dot{\delta q_{2}} & =\frac{p_{1}}{q_{1}} \delta q_{2}+\frac{\delta l_{3}}{q_{1}} \\
& =d_{t}\left(\log q_{1}\right) \delta q_{2}+\frac{\delta l_{3}}{q_{1}} .
\end{aligned}
$$


Particular and homogeneous solutions are given by $\delta l_{3} q_{1} \int q_{1}^{-2} d t$ and $q_{1}$ respectively. The homogeneous solution, $\boldsymbol{\delta} \mathbf{q}=\left(0, q_{1}, 0\right)$, already enters (36) in the form $\mathbf{k} \wedge \mathbf{q}$ and the particular solution will therefore suffice. By assuming $\boldsymbol{\delta} \mathbf{q}=\left(0,0, \delta q_{3}\right)$ we similarly obtain the solution $\delta q_{3}=\delta l_{2} q_{1} \int q_{1}^{-2} d t$. We have:

$$
\begin{aligned}
\boldsymbol{\delta} \mathbf{q} & =-\mathbf{p} \delta t_{0}-\mathbf{k} \wedge \mathbf{q} \delta \epsilon_{2}+\mathbf{j} \wedge \mathbf{q} \delta \epsilon_{3}+\left(\mathbf{q}-\frac{3}{2} \mathbf{p}\left(t-t_{0}\right)\right) \frac{-\delta h}{h} \\
& +\delta l_{3}\left(0, q_{1} \int q_{1}^{-2} d t, 0\right)+\delta l_{2}\left(0,0, q_{1} \int q_{1}^{-2} d t\right)
\end{aligned}
$$

For $h=0$ we may explicitly solve the reduced equations for $\mathbf{q}=\left(q_{1}, 0,0\right), \mathbf{p}=\left(\dot{q}_{1}, 0,0\right)$ :

$$
\dot{q}_{1}^{2}-\frac{2 \mu}{q_{1}}=0
$$

by separation of variable to obtain:

$$
q_{1}(t)=\left(q_{1}\left(t_{0}\right) \pm \frac{3}{2} \sqrt{2 \mu}\left(t-t_{0}\right)\right)^{2 / 3} .
$$

This only exists for $t-t_{0} \in\left(-\frac{2}{3 \sqrt{2 \mu}} q_{1}\left(t_{0}\right), \infty\right)$ and $t-t_{0} \in\left(-\infty, \frac{2}{3 \sqrt{2 \mu}} q_{1}\left(t_{0}\right)\right)$. For radially expanding and contracting orbits we take + and - in (39) respectively. From this we realise that the two variational solutions:

$$
\left(\mathbf{q}-\frac{3}{2} \mathbf{p}\left(t-t_{0}\right)\right)=\frac{2 q_{1}\left(t_{0}\right)}{\left( \pm 12 \sqrt{2 \mu}\left(t-t_{0}\right)+8 q_{1}\left(t_{0}\right)\right)^{1 / 3}} \mathbf{i}
$$

and

$$
\mathbf{p}=\frac{2 \sqrt{2} \mu}{\left( \pm 12 \sqrt{2 \mu}\left(t-t_{0}\right)+8 q_{1}\left(t_{0}\right)\right)^{1 / 3}} \mathbf{i},
$$

are proportional to each other. To obtain a sixth linearly independent solution we solve (11) for the conservation of $\delta h$ :

$$
\begin{aligned}
\delta h & =p_{1} \delta p_{1}+\frac{\mu}{q_{1}^{2}} \delta q_{1} \\
\dot{\delta q_{1}} & =-\frac{\mu}{p_{1} q_{1}^{2}} \delta q_{1}+\frac{\delta h}{p_{1}} \\
& =\frac{\dot{p}_{1}}{p_{1}} \delta q_{1}+\frac{\delta h}{p_{1}} \\
& =d_{t}\left(\log \left(p_{1}\right)\right) \delta q_{1}+\frac{\delta h}{p_{1}} .
\end{aligned}
$$


The homogeneous solution space is spanned by $\delta q_{1}=p_{1}$ and by separation we obtain an inhomogeneous solution:

$$
\delta q_{1}=p_{1} \int p_{1}^{-2} d t \delta h=\frac{1}{80 \mu}\left(12 \sqrt{2 \mu}\left(t-t_{0}\right)+8 q_{1}\left(t_{0}\right)\right)^{4 / 3} \delta h .
$$

\subsection{Summary of results}

We are ready to collect the conclusions:

Main result The complete solution of the variational equations of Kepler's problem for $\epsilon \neq 0,1$ with

$$
\begin{aligned}
\boldsymbol{q} & =\frac{l^{2} / \mu}{1+\epsilon \cos \nu} \cos \nu \boldsymbol{i}+\frac{l^{2} / \mu}{1+\epsilon \cos \nu} \sin \nu \boldsymbol{j}, \\
\boldsymbol{p} & =-\frac{\mu}{l} \sin \nu \boldsymbol{i}+\frac{\mu}{l}(\epsilon+\cos \nu) \boldsymbol{j}
\end{aligned}
$$

$i s$

$$
\begin{aligned}
\boldsymbol{\delta} \boldsymbol{q}\left(t ; \delta t_{0}, \boldsymbol{\delta} \boldsymbol{\epsilon}, \boldsymbol{\delta} \boldsymbol{l}, \delta h\right) & =-\boldsymbol{p} \delta t_{0}+\boldsymbol{k} \wedge \boldsymbol{q} \frac{\delta \epsilon_{2}}{\epsilon}+\boldsymbol{j} \wedge \boldsymbol{q} \frac{\delta l_{1}}{l}+\boldsymbol{i} \wedge \boldsymbol{q} \frac{\delta l_{2}}{l} \\
& +\left(\boldsymbol{q}-\frac{3}{2} \boldsymbol{p}\left(t-t_{0}\right)\right) \frac{-\delta h}{h}-\left(\frac{l^{2}}{\mu} \boldsymbol{i}+\sin \nu \boldsymbol{q} \wedge \boldsymbol{k}\right) \frac{1}{1-\epsilon^{2}} \delta \epsilon_{1}
\end{aligned}
$$

which lifts to

$$
\begin{array}{r}
\boldsymbol{\delta} \boldsymbol{p}\left(t ; \delta t_{0}, \boldsymbol{\delta} \boldsymbol{\epsilon}, \boldsymbol{\delta} \boldsymbol{l}, \delta h\right)=\frac{\mu}{q^{3}} \boldsymbol{q} \delta t_{0}+\boldsymbol{k} \wedge \boldsymbol{p} \frac{\delta \epsilon_{2}}{\epsilon}+\boldsymbol{j} \wedge \boldsymbol{p} \frac{\delta l_{1}}{l}+\boldsymbol{i} \wedge \boldsymbol{p} \frac{\delta l_{2}}{l} \\
-\frac{1}{2}\left(\boldsymbol{p}-3 \frac{\mu}{q^{3}} \boldsymbol{q}\left(t-t_{0}\right)\right) \frac{-\delta h}{h}-\left(\sin \nu \boldsymbol{p} \wedge \boldsymbol{k}+\mu^{2} / l^{3}(1+\epsilon \cos \nu)^{2} \cos \nu \boldsymbol{q} \wedge \boldsymbol{k}\right) \frac{1}{1-\epsilon^{2}} \delta \epsilon_{1}
\end{array}
$$

where the subscripts 1,2 and 3 refer to the coordinates of $\boldsymbol{i}=\frac{\boldsymbol{\epsilon}}{e}, \boldsymbol{j}=\frac{\boldsymbol{\epsilon} \wedge \boldsymbol{l}}{\epsilon \wedge l}$ and $\boldsymbol{k}=\frac{\boldsymbol{l}}{l}$ respectively. 
For a circular reference orbit $(\epsilon=0)$ :

$$
\begin{aligned}
\boldsymbol{\delta} \boldsymbol{q} & =-\boldsymbol{p} \delta t_{0}+\frac{\boldsymbol{\lambda} \wedge \boldsymbol{q}}{l} \delta l_{1}+\frac{\boldsymbol{\iota} \wedge \boldsymbol{q}}{l} \delta l_{2}+\left(\boldsymbol{q}-\frac{3}{2} \boldsymbol{p}\left(t-t_{0}\right)\right) \frac{-\delta h}{h} \\
& +l^{2} / \mu\left(-\left(1+\sin ^{2} \nu\right) \delta \epsilon_{1}+\cos \nu \sin \nu \delta \epsilon_{2}\right) \boldsymbol{\iota} \\
& +l^{2} / \mu\left(\cos \nu \sin \nu \delta \epsilon_{1}-\left(1+\cos ^{2} \nu\right) \delta \epsilon_{2}\right) \boldsymbol{\lambda} \\
\boldsymbol{\delta} \boldsymbol{p} & =\frac{\mu}{q^{3}} \boldsymbol{q} \delta t_{0}+\frac{\boldsymbol{\lambda} \wedge \boldsymbol{p}}{l} \delta l_{1}+\frac{\boldsymbol{\iota} \wedge \boldsymbol{p}}{l} \delta l_{2}-\frac{1}{2}\left(\boldsymbol{p}-3 \frac{\mu}{q^{3}} \boldsymbol{q}\left(t-t_{0}\right)\right) \frac{-\delta h}{h} \\
& +\mu^{2} / l(1+\epsilon \cos \nu)^{2}\left(-2 \cos \nu \sin \nu \delta \epsilon_{1}+\left(\cos ^{2} \nu-\sin ^{2} \nu\right) \delta \epsilon_{2}\right) \boldsymbol{\iota} \\
& +\mu^{2} / l(1+\epsilon \cos \nu)^{2}\left(\left(\cos ^{2} \nu-\sin ^{2} \nu\right) \delta \epsilon_{1}+2 \cos \nu \sin \nu \delta \epsilon_{2}\right) \boldsymbol{\lambda}
\end{aligned}
$$

where $(\boldsymbol{\iota}, \boldsymbol{\lambda}) \subset P$ is any orthonormal basis in the orbital plane and subscripts 1 and 2 now refer to coordinates with respect to $\iota$ and $\boldsymbol{\lambda}$ respectively.

For a parabolic reference orbit where $\epsilon=1, l \neq 0$ and $h=0$ :

$$
\begin{aligned}
\boldsymbol{\delta} \boldsymbol{q} & =-\boldsymbol{p} \delta t_{0}+\boldsymbol{k} \wedge \boldsymbol{q} \delta \epsilon_{2}+\boldsymbol{j} \wedge \boldsymbol{q} \frac{\delta l_{1}}{l}+\boldsymbol{i} \wedge \boldsymbol{q} \frac{\delta l_{2}}{l} \\
& +2\left(\boldsymbol{q}-\frac{3}{2} \boldsymbol{p}\left(t-t_{0}\right)\right) \frac{\delta l_{3}}{l} \\
& +\frac{2 l^{4} / \mu^{3}}{5(1+\cos \nu)^{2}}\left(2+4 \cos \nu-9 \cos ^{2} \nu-2 \cos ^{3} \nu\right) \boldsymbol{i} \delta h \\
& -\frac{2 l^{4} / \mu^{3}}{5(1+\cos \nu)^{2}} \sin \nu\left(11 \cos \nu+2\left(1+\cos ^{2} \nu\right)\right) \boldsymbol{j} \delta h, \\
\boldsymbol{\delta} \boldsymbol{p} & =\frac{\mu}{q^{3}} \boldsymbol{q} \delta t_{0}+\boldsymbol{k} \wedge \boldsymbol{p} \frac{\delta \epsilon_{2}}{\epsilon}+\boldsymbol{j} \wedge \boldsymbol{p} \frac{\delta l_{1}}{l}+\boldsymbol{i} \wedge \boldsymbol{p} \frac{\delta l_{2}}{l} \\
& -\frac{1}{2}\left(\boldsymbol{p}-3 \frac{\mu}{q^{3}} \boldsymbol{q}\left(t-t_{0}\right)\right) \frac{\delta l_{3}}{l}+ \\
& +\frac{2 l}{5 \mu(1+\cos \nu)} \sin \nu \cos \nu\left(1+3 \cos \nu+\cos ^{2} \nu\right) \boldsymbol{i} \delta h \\
& +\frac{l}{5 \mu}\left(7-2 \cos \nu\left(8+2 \cos \nu+\cos ^{2} \nu\right)\right) \boldsymbol{j} \delta h .
\end{aligned}
$$


For a purely radial reference orbit where $\epsilon=1, l=0$ and $h \neq 0$ :

$$
\begin{aligned}
\boldsymbol{\delta} \boldsymbol{q} & =-\boldsymbol{p} \delta t_{0}-\boldsymbol{k} \wedge \boldsymbol{q} \delta \epsilon_{2}+\boldsymbol{j} \wedge \boldsymbol{q} \delta \epsilon_{3}+\left(\boldsymbol{q}-\frac{3}{2} \boldsymbol{p}\left(t-t_{0}\right)\right) \frac{-\delta h}{h} \\
& +\delta l_{3}\left(0, q_{1} \int q_{1}^{-2} d t, 0\right)+\delta l_{2}\left(0,0, q_{1} \int q_{1}^{-2} d t\right) \\
\boldsymbol{\delta} \boldsymbol{p} & =\frac{\mu}{q^{3}} \boldsymbol{q} \delta t_{0}-\boldsymbol{k} \wedge \boldsymbol{p} \delta \epsilon_{2}+\boldsymbol{j} \wedge \boldsymbol{p} \delta \epsilon_{3}-\frac{1}{2}\left(\boldsymbol{p}-3 \frac{\mu}{q^{3}} \boldsymbol{q}\left(t-t_{0}\right)\right) \frac{-\delta h}{h} \\
& +\delta l_{3}\left(0, p_{1} \int q_{1}^{-2} d t+\frac{1}{q_{1}}, 0\right)+\delta l_{2}\left(0,0, p_{1} \int q_{1}^{-2} d t+\frac{1}{q_{1}}\right)
\end{aligned}
$$

When $\epsilon=1, l=0$ and $h=0$ then $\left(\boldsymbol{q}-\frac{3}{2} \boldsymbol{p}\left(t-t_{0}\right)\right) \frac{-\delta h}{h}$ in (49) is replaced with $\delta h\left(p_{1} \int p_{1}^{-2} d t, 0,0\right)$, $\int p_{1}^{-2} d t=\frac{1}{80 \mu}\left( \pm 12 \sqrt{2 \mu}\left(t-t_{0}\right)+8 q_{1}\left(t_{0}\right)\right)^{4 / 3}$ where + and - refer to radially expanding and contracting reference solutions respectively.

For $\epsilon \neq 1$ the solution is in agreement with the solutions obtained by Palmer and Imre in [16] (see equations (41)-(44) and note that $A$ and $B$ are related to the conserved quantities through: $A=-\mu^{2} \delta t_{0} / l^{3}$ and $\left.B=\delta \epsilon_{2} / \epsilon\right)$.

As expected, and discussed in section 2, only the variation in $h$ gives rise to unbounded relative motion for $\epsilon<1$. Obviously, the unboundedness is not physical and is only a result of our linearisation. An illustration of the effects of the variations in the six quantities $t_{0}, l_{1}$, $l_{2}, \epsilon_{2}, \epsilon_{1}$ and $h$ in terms of conic sections is seen in Fig. 3 for $\epsilon<1$. For $\epsilon \geq 1$ the reference trajectory $(\mathbf{q}, \mathbf{p})$ is no longer periodic, in fact $\mathbf{q}(t) \rightarrow \infty$ as $t \rightarrow \infty$ for $\epsilon>1$ and $\epsilon=1$ with $h \geq 0$. Therefore, the variational solutions around hyperbolic and parabolic orbits may grow unbounded even if $\delta h=0$. Moreover, the implication of infinitesimal difference in energies for the case of a parabolic and hyperbolic reference is no longer straightforward. For parabolic and hyperbolic orbits the velocities converge to $\mathbf{0}$ or a constant $\pm \mathbf{v}_{\infty}$ respectively as $t \rightarrow \pm \infty$, cf. e.g. (42). Hence $\mathbf{q}-\frac{3}{2} \mathbf{p}\left(t-t_{0}\right)$, the variational solution corresponding to variations in $h$, can either approach $\pm \infty$ or 0 when $t \rightarrow \pm \infty$.

For sufficiently large $t$ we may neglect the azimuthal variation since $\dot{\nu} \rightarrow 0$ for $\nu \rightarrow \nu^{*}$, where $\nu^{*}$ is such that $1+\epsilon \cos \nu^{*}=0$, and the drift to infinity is asymptotically radial. 
Similarly for $t \rightarrow-\infty$. For $h=0$ it then directly follows from (40) that

$$
q-\frac{3}{2} p\left(t-t_{0}\right):=\left|\mathbf{q}-\frac{3}{2} \mathbf{p}\left(t-t_{0}\right)\right| \rightarrow 0, \quad \text { for } \quad t \rightarrow \infty, h=0,
$$

for $t \rightarrow \pm \infty$. For $h>0$, $\lim _{t \rightarrow \infty} \dot{q}=v_{\infty}=\sqrt{2 h}<\infty$ and hence $q-\sqrt{2 h} t \rightarrow$ const. for $t \rightarrow \infty$, so that

$$
\left|\left(q-\frac{3}{2} p\left(t-t_{0}\right)\right) \frac{-\delta h}{h}\right|-\frac{|\delta h|}{\sqrt{2 h}} t \rightarrow \pm \text { const. } \quad \text { for } \quad t \rightarrow \infty, h>0, \delta h \neq 0 .
$$

It follows that only for parabolic reference orbits will neighbouring satellites, whose orbits are only separated by an infinitesimal difference in energy, catch up at infinity.

In the following section we shall demonstrate the usefulness of having solutions that are easily interpreted geometrically in the design of formation flying trajectories.

\section{$5 \quad$ Design of formation flying trajectories}

We construct orbits for four satellites so they in position space form a regular tetrahedral formation. Any inter-satellite formation will obviously, at least in general, be distorted over the period of the orbit. The aim is therefore to obtain the tetrahedral formation at a certain stage of the orbit and, in the theme of the paper, it will be constructed by determining the required values of the conserved quantities. An application might be a mission orbiting the Sun to visit the Kuiper belt.

The Cluster mission II is currently flying and operating successfully in its ninth year exploiting the benefits of a tetrahedral formation. Furthermore, several future missions are planning to use such formations. This interest is due to the fact that the tetrahedral formations allow for resolving three dimensional gradients, for example in the context of space weather and environment. This mission scenario therefore provides a realistic example where the use of our solutions can be demonstrated.

We shall choose our formation so that the four satellites are all at aphelion when at the Kuiper belt and, perhaps most importantly, all reach their aphelion at the same time. The 


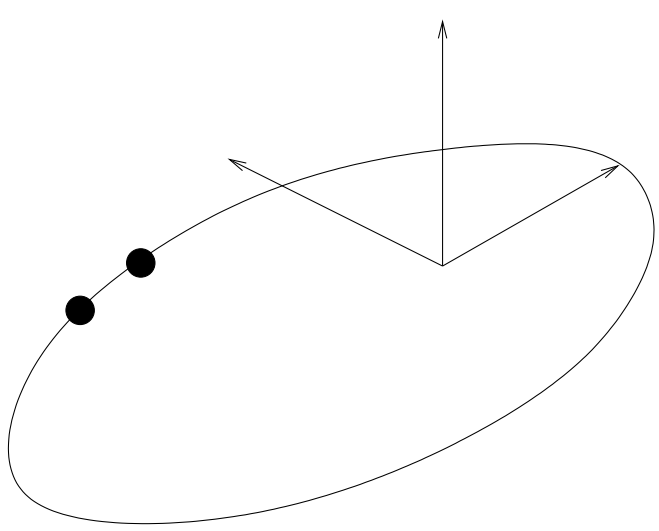

(a) Variation in $t_{0}$ : satellites on same conic section but with a delay

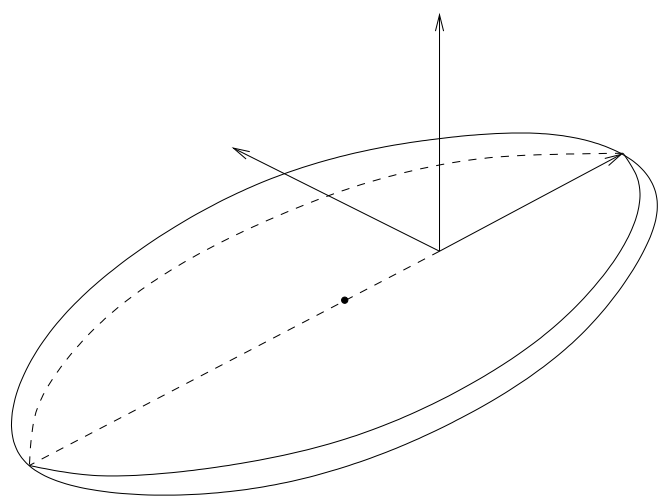

(c) Variations in $l_{2}$ : rotation of orbital plane about $\epsilon$

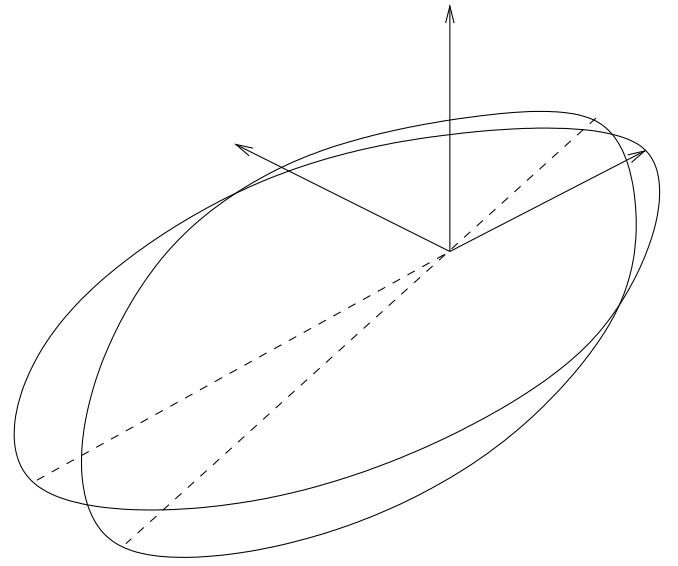

(e) Variations in $\epsilon_{2}$ : rotation of conic section about $\mathbf{l}$

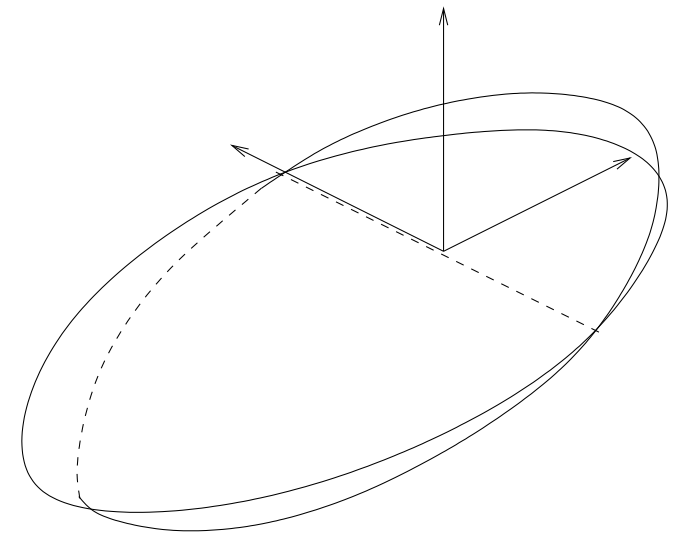

(b) Variations in $l_{1}$ : rotation of orbital plane about $\mathbf{l} \wedge \boldsymbol{\epsilon}$

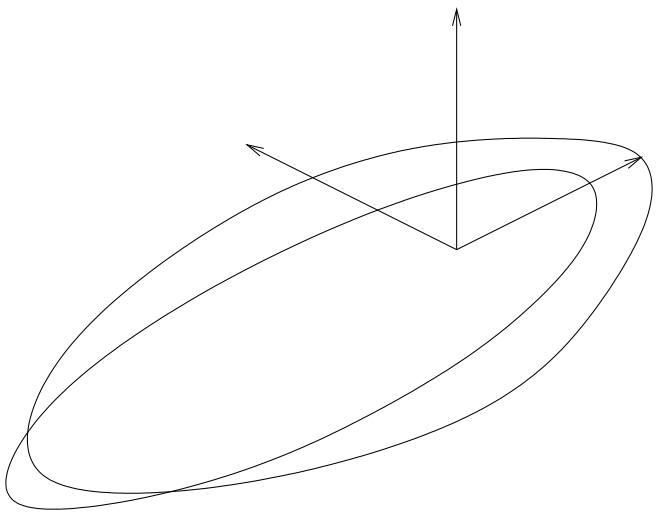

(d) Variations in $\epsilon_{1}$ : changing magnitude of $\epsilon$

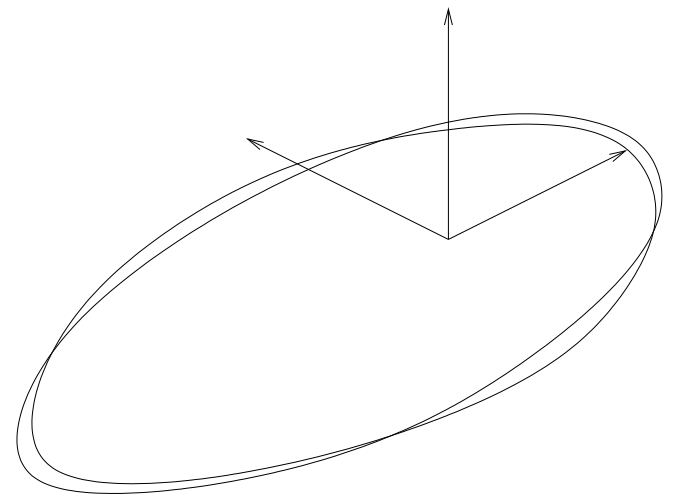

(f) Variations in $h$ : changing semi-major axis

Figure 3: An illustration of the effects of the six independent variations in terms of conic sections for $\epsilon<1$. The first five result in bounded relative motion whereas variation in energy results in unbounded solutions of variational equations in accordance to Kepler's third law. 
former will maximise the duration of the formation, whereas the latter is enforced by $\delta t_{0}=0$ and fixing the energy $h$ or, equivalently, the semi-major axis a cf. (4). Note, however, that $\delta t_{0} \neq 0, \delta h \neq 0$ would allow for different injection times which in certain applications might be useful.

Changing $l_{2}$, within the first order approximation, does not change anything at aphelion and perihelion, see e.g. Fig. 3, and therefore we may, at least initially, set $\delta l_{2}=0$. We are left with three variations $\delta \epsilon_{1}, \delta \epsilon_{2}$ and $\delta l_{1}$, which respectively have the effect of changing the magnitude of the eccentricity, changing the orientation of the conic section within the orbital plane, and rotating the orbital plane about $\mathbf{j}$, the direction of semilatus rectum. For $\delta t_{0}=0, \delta h=0$ and $\delta l_{2}=0(43)$ reads

$$
\boldsymbol{\delta} \mathbf{q}=\mathbf{k} \wedge \mathbf{q} \frac{\delta \epsilon_{2}}{\epsilon}+\mathbf{j} \wedge \mathbf{q} \frac{\delta l_{1}}{l}-\left(\frac{l^{2}}{\mu} \mathbf{i}+\sin \nu \mathbf{q} \wedge \mathbf{k}\right) \frac{1}{1-\epsilon^{2}} \delta \epsilon_{1}
$$

In particular at aphelion:

$$
\left.\boldsymbol{\delta} \mathbf{q}\right|_{\nu=\pi}=-a \delta \epsilon_{1} \mathbf{i}-a(1+\epsilon) \frac{\delta \epsilon_{2}}{\epsilon} \mathbf{j}+a(1+\epsilon) \frac{\delta l_{1}}{l} \mathbf{k}
$$

where we have made use of $l^{2} / \mu=a\left(1-\epsilon^{2}\right)$.

The configuration of the regular tetrahedron at aphelion is given by a centre point and a rotation matrix $\mathbf{R}_{\alpha, \beta, \gamma}$ mapping the inertial frame to a body fixed frame: $\mathbf{R}_{\alpha, \beta, \gamma}:(\mathbf{i}, \mathbf{j}, \mathbf{k}) \mapsto$ $\left(\mathbf{i}^{\prime}, \mathbf{j}^{\prime}, \mathbf{k}^{\prime}\right)$. We let the reference orbit pass through the centre point and let $\mathbf{R}_{\alpha, \beta, \gamma}$ depend on three Euler angles $\alpha, \beta$ and $\gamma$. As illustrated in Fig. 4, $\alpha$ is the angle between $\mathbf{i}$ and the line of nodes $\mathbf{n}, \beta$ is the angle between $\mathbf{k}$ and $\mathbf{k}^{\prime}$ and finally $\gamma$ is the angle between the line of nodes and $\mathbf{i}^{\prime}$. With these definitions $\mathbf{R}_{\alpha, \beta, \gamma}$ reads:

$$
\mathbf{R}_{\alpha, \beta, \gamma}=\left(\begin{array}{ccc}
c_{\alpha} c_{\gamma}-s_{\alpha} c_{\beta} s_{\gamma} & -c_{\alpha} s_{\gamma}-s_{\alpha} c_{\beta} c_{\gamma} & s_{\beta} s_{\alpha} \\
s_{\alpha} c_{\gamma}+c_{\alpha} c_{\beta} s_{\gamma} & -s_{\alpha} s_{\gamma}+c_{\alpha} c_{\beta} c_{\gamma} & -s_{\beta} c_{\alpha} \\
s_{\beta} s_{\gamma} & s_{\beta} c_{\gamma} & c_{\beta}
\end{array}\right)
$$

where we have used the usual compact notation: $s_{v}=\sin v$ and $c_{v}=\cos v$ for every $v$.

In Fig. 5 the tetrahedron is shown in the body frame $\left(\mathbf{i}^{\prime}, \mathbf{j}^{\prime}, \mathbf{k}^{\prime}\right)$. We let $\mathbf{k}^{\prime}$ be directed through one of the vertices, which we call $\mathbf{1}$. The axes $\mathbf{i}^{\prime}$ and $\mathbf{j}^{\prime}$ are defined so that $\mathbf{2}$ 's 


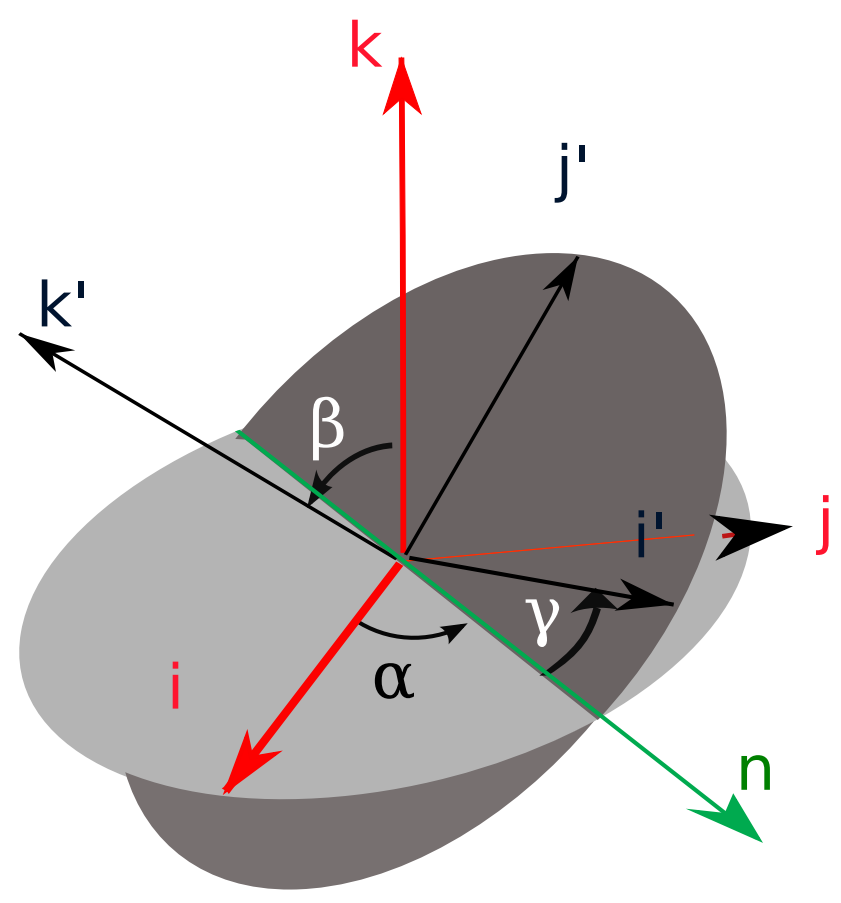

Figure 4: Euler angles.

projection onto the plane

$$
\left\{\mathbf{x} \in \mathbb{R}^{3} \mid\left\langle\mathbf{x}, \mathbf{k}^{\prime}\right\rangle=0\right\}
$$

equals $c \mathbf{i}^{\prime}$ for $c=\cos \left(\pi / 2-\theta^{*}\right)$, where $\theta^{*}=\arccos (-1 / 3) \approx 109.5^{\circ}$ is the angle between the segments joining the centre with the vertices. Here $\mathbf{3}$ is the vertex located a positive $2 \pi / 3$, i.e. $120^{\circ}$, rotation about $\mathbf{k}^{\prime}$ from $\mathbf{2}$. The four satellites are coloured respectively black, green, red and cyan in Fig. 5. Straightforward geometry shows that the distance from the tetrahedron centre to the vertices is $R=\sqrt{3 / 8} \ell$, where $\ell$ is the side-length.

We let the satellites, $\mathbf{1}, \mathbf{2}, \mathbf{3}$ and $\mathbf{4}$, be positioned at $\boldsymbol{\delta} \mathbf{q}^{(i)}, i=1,2,3$ and 4 from the centre 


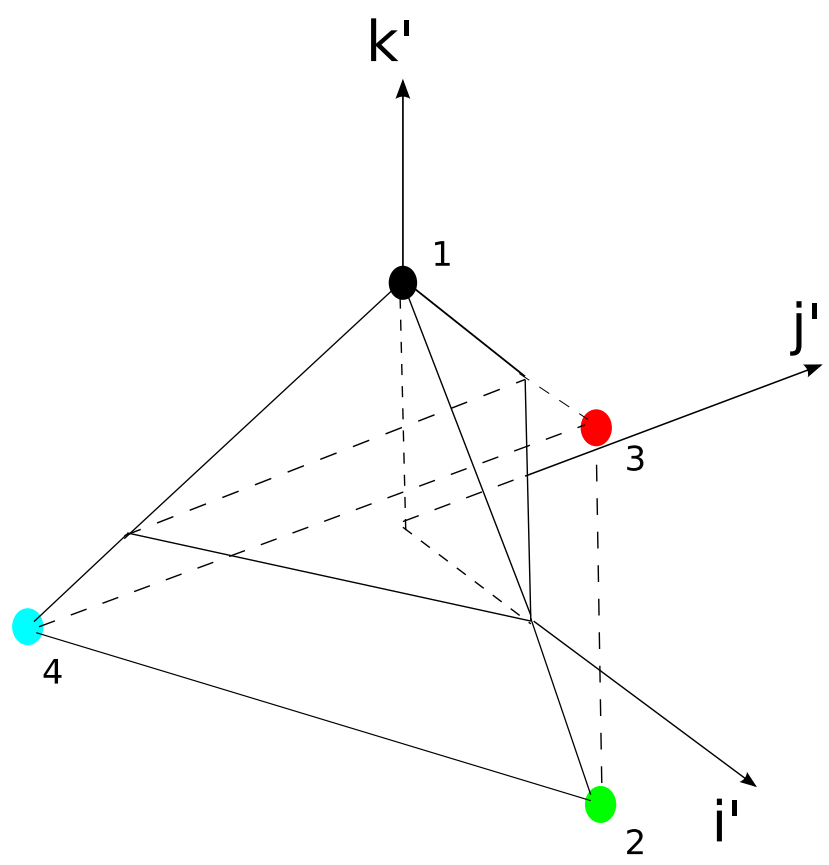

Figure 5: The tetrahedron in the body frame. The four satellites, 1, 2, 3 and 4, are coloured respectively black, green, red and cyan.

point. Then in the body frame we have:

$$
\begin{aligned}
& \boldsymbol{\delta} \mathbf{q}^{(1)}=R \mathbf{k}^{\prime}, \\
& \boldsymbol{\delta} \mathbf{q}^{(2)}=R\left(\sin \theta^{*} \mathbf{i}^{\prime}+\cos \theta^{*} \mathbf{k}^{\prime}\right), \\
& \delta \mathbf{q}^{(3)}=R\left(-\frac{1}{2} \sin \theta^{*} \mathbf{i}^{\prime}+\frac{\sqrt{3}}{2} \sin \theta^{*} \mathbf{j}^{\prime}+\cos \theta^{*} \mathbf{k}^{\prime}\right), \\
& \delta \mathbf{q}^{(4)}=R\left(-\frac{1}{2} \sin \theta^{*} \mathbf{i}^{\prime}-\frac{\sqrt{3}}{2} \sin \theta^{*} \mathbf{j}^{\prime}+\cos \theta^{*} \mathbf{k}^{\prime}\right),
\end{aligned}
$$

which may be related to the inertial frame through $\mathbf{R}_{\alpha, \beta, \gamma}$.

Using (51) we obtain the following equations for the formation to have the configuration specified by $\mathbf{R}_{\alpha, \beta, \gamma}$ at aphelion:

$$
\begin{aligned}
\left\langle\boldsymbol{\delta} \mathbf{q}^{(i)}, \mathbf{i}\right\rangle & =-a \delta \epsilon_{1}^{(i)} \\
\left\langle\boldsymbol{\delta} \mathbf{q}^{(i)}, \mathbf{j}\right\rangle & =-a(1+\epsilon) \frac{\delta \epsilon_{2}^{(i)}}{\epsilon} \\
\left\langle\boldsymbol{\delta} \mathbf{q}^{(i)}, \mathbf{k}\right\rangle & =a(1+\epsilon) \frac{\delta l_{1}^{(i)}}{l}
\end{aligned}
$$


for $i=1,2,3$ and 4 , where $\delta l_{1}^{(i)}, \delta \epsilon_{1}^{(i)}, \delta \epsilon_{2}^{(i)}$ are the variations in the three constants of motion for satellite $i$. Solving the linear equations gives

$$
\begin{aligned}
\delta \epsilon_{1}^{(i)} & =-\frac{1}{a}\left\langle\boldsymbol{\delta} \mathbf{q}^{(i)}, \mathbf{i}\right\rangle, \\
\delta \epsilon_{2}^{(i)} & =-\frac{\epsilon}{a(1+\epsilon)}\left\langle\boldsymbol{\delta} \mathbf{q}^{(i)}, \mathbf{j}\right\rangle, \\
\delta l_{1}^{(i)} & =\frac{l}{a(1+\epsilon)}\left\langle\boldsymbol{\delta} \mathbf{q}^{(i)}, \mathbf{k}\right\rangle .
\end{aligned}
$$

In Fig. 6, the evolution of a formation is visualised. Here we have for simplicity chosen $\mathbf{R}_{\alpha, \beta, \gamma}=\mathbf{I}$, and:

$$
\begin{aligned}
& \delta \epsilon_{1}^{(1)}=0, \quad \delta \epsilon_{2}^{(1)}=0, \quad \delta l_{1}^{(1)}=\frac{R l}{a(1+\epsilon)}, \\
& \delta \epsilon_{1}^{(2)}=-\frac{R \sin \theta^{*}}{a}, \delta \epsilon_{2}^{(2)}=0, \quad \delta l_{1}^{(2)}=\frac{R l \cos \theta^{*}}{a(1+\epsilon)}, \\
& \delta \epsilon_{1}^{(3)}=\frac{R \sin \theta^{*}}{2 a}, \quad \delta \epsilon_{2}^{(3)}=-\frac{\sqrt{3} \epsilon R \sin \theta^{*}}{2 a(1+\epsilon)}, \quad \delta l_{1}^{(3)}=\frac{R l \cos \theta^{*}}{a(1+\epsilon)}, \\
& \delta \epsilon_{1}^{(4)}=\frac{R \sin \theta^{*}}{2 a}, \quad \delta \epsilon_{2}^{(4)}=\frac{\sqrt{3} \epsilon R \sin \theta^{*}}{2 a(1+\epsilon)}, \quad \delta l_{1}^{(4)}=\frac{R l \cos \theta^{*}}{a(1+\epsilon)} .
\end{aligned}
$$

The units on the axes in Fig. 6 are such that 1 equals the semi-major axis. We have set $\epsilon=\frac{1}{2}$ and the side length of the tetrahedron to $\ell=\frac{1}{2}$ which corresponds to half the semi-major axis and is obviously physically unreasonable, but it is convenient in terms of visualisation.

As expected, the formation is distorted as the satellites move away from aphelion. At perihelion the formation has, when compared to aphelion, been slightly distorted and reflected about the orbital plane. At semilatus rectum, the satellites are all in the same orbital plane, which is also in agreement with Fig. 3. By varying $l_{2}$ one may move the satellites out of this common plane, see e.g. Fig. 3. This variation will not change the configuration at aphelion and perihelion. 


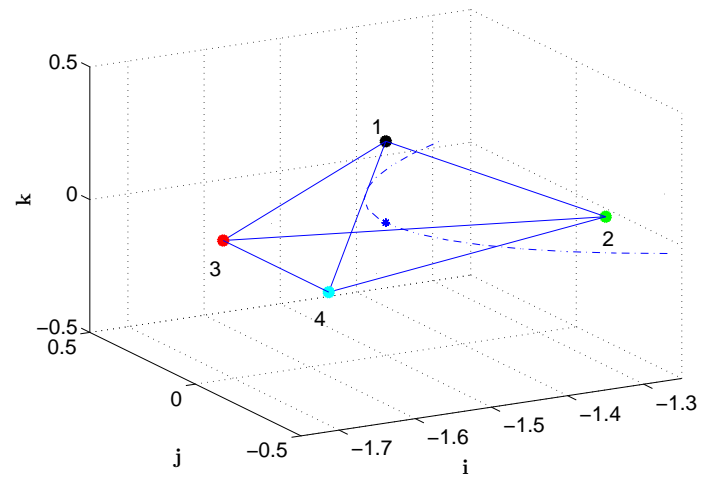

(a) Satellite formation at aphelion.

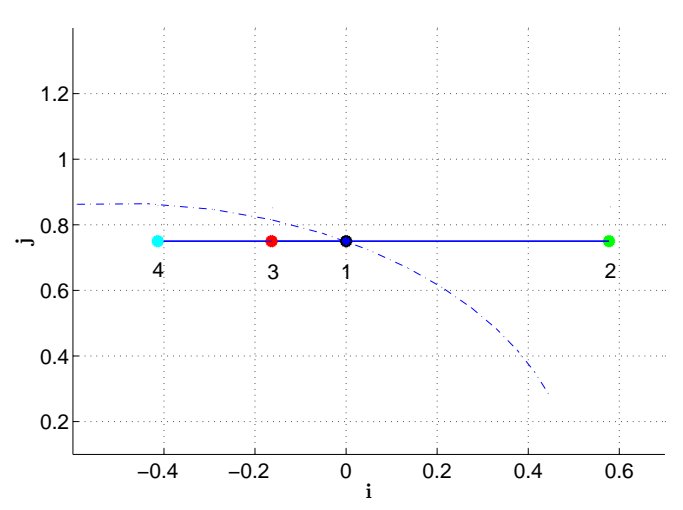

(b) Satellite formation at semilatus rectum.

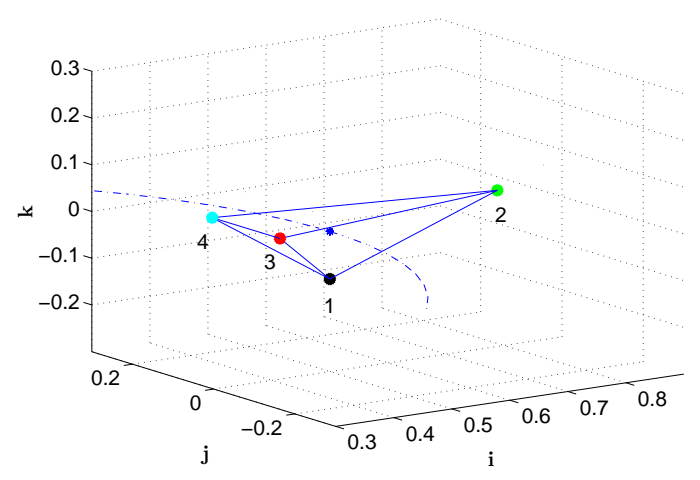

(c) Satellite formation at perihelion.

Figure 6: A visualisation of a formation near an elliptic orbit. The formation is designed to be a regular tetrahedron at aphelion. The formation is distorted as it moves around the Sun and they will all be in the same orbital plane at semilatus rectum.

\section{Discussion on perturbations}

The approach developed rely on the super-integrability of the Kepler problem. Any perturbation will in general destroy the symmetries and the integrability, let alone the super- 
integrability. Particularly, the formation dynamics predicted by the solution above will eventually differ significantly from the true formation. However, the net effect of perturbations depends on the size of the perturbation and also on time scales. Therefore, the solutions may in fact be a good approximation to the true formation dynamics near certain reference trajectories over a large period of time. For example, the effect of the oblateness of the Earth decreases as $q$ increases as $q^{-4}$ and is therefore primarily significant for near Earth orbiting formations. Moreover, for highly eccentric orbits, parabolic or hyperbolic orbits this effect is only important through the short duration flight through perigee such that a significant effect may not even be expected on a time scale of many orbital periods. In this latter scenario the third body effects might obviously contribute with more important perturbations.

Several references account for the oblateness of the Earth, see [5, 9, 11, 17, 18, 25]. For example in [11] this perturbation was included in a numerical formation propagator by separating the solution into the analytical Keplerian part (11) and a sympletic numerical part.

\section{Conclusion}

We obtained analytical solutions of the variational equation of the Kepler problem about any reference orbit in a compact form by relying on the super-integrability of the Kepler problem. The solutions were written in terms of the relevant conserved quantities: relative energy, relative angular momentum and relative eccentricity vector and the geometrical setting, in which the solutions were derived, allowed for a straightforward design of tetrahedral formations on highly eccentric orbits.

\section{Acknowledgement}

This work was supported by EU funding for the Marie-Curie Research Training Network AstroNet (contract number: MRTN-CT-2006-035151). 


\section{References}

[1] R.A. Broucke. Solution of the elliptic rendezvous problem with the time as independent variable. Journal of Guidance, Control and Dynamics, 26(4):615-621, 2003.

[2] W.H. Clohessy and R.S. Wiltshire. Terminal guidance system for satellite rendezvous. Journal of the Aerospace Sciences, 27(9):653-658, 674, 1960.

[3] R. H. Cushmann and L. M. Bates. Global Aspects of Classical Integrable Systems. Birkhauser Verlag: Basel, Boston and Berlin, 1997.

[4] C. Ph. Escoubet. Cluster-ii: Scientific objectives and data dissemination. Technical report, ESA, ESTEC, Noordwijk, The Netherlands, 2000.

[5] Giancarmine Fasano and Marco D'Errico. Modelling orbital relative motion to enable formation design from application requirements. Journal of Celestial Mechanics and Dynamical Astronomy, 105:113-139, 2009.

[6] D. Folta, J. Bristow, A. Hawkins, and G. Dell. Enhanced formation flying validation report (gsfc algorithm). Technical report, NASA/GSFC, Maryland, USA, 2002.

[7] G. Gómez and M. Marcote. High-order analytical solutions of Hill's equations. Journal of Celestial Mechanics and Dynamical Astronomy, 94:197-211, 2006.

[8] J.R. Guinn. Enhanced Formation Flying Validation Report (JPL Algorithm). Technical report, NASA/GSFC, Maryland, USA, 2002.

[9] M. Halsall and P.L. Palmer. Modelling natural formations of leo satellites. Journal of Celestial Mechanics and Dynamical Astronomy, 99:105-127, 2007.

[10] G. W. Hill. Researches in the lunar theory. American Journal of Mathematics, 1:5-26, 129-147, 245-260, 1878. 
[11] E. Imre and P.L. Palmer. High precission symplectic numerical relative orbit propagation. Journal of Guidance, Control and Dynamics, 4:965-973, 2007.

[12] C.D. Karlgaard and F.H. Lutze. Second-order relative motion equations. Journal of Guidance, Control and Dynamics, 26(1):41-49, 2003.

[13] D.F. Lawden. Fundamentals of space navigation. Journal of Britsh Interplanetary Society, 13:87-101, 1954.

[14] J. E. Marsden, T. Ratiu, and G. Raugel. Symplectic connections and the linearisation of hamiltonian systems. Proceedings of Royal Society of Edinburgh, 117A:329-380, 1991.

[15] R.G. Melton. Time-explicit representation of relative motion between elliptical orbits. Journal of Guidance, Control and Dynamics, 23(4):604-610, 2000.

[16] P.L. Palmer and E. Imre. Relative motion of satellites on neighbouring keplerian orbits. Journal of Guidance, Control, and Dynamics, 30(2):521-528, 2007.

[17] H. Schaub and K. T. Alfriend. $J_{2}$ invariant relative orbits for spacecraft formations. Journal of Celestial Mechanics and Dynamical Astronomy, 79:77-95, February 2001.

[18] S.A. Schweighart and R.J. Sedwick. High-fidelity linearized $J_{2}$ model for satellite formation flight. Journal of Guidance, Control and Dynamics, 25(6):1073-1080, 2002.

[19] Prasenjit Sengupta, Srinivas R. Vadali, and Kyle T. Alfriend. Second-order state transition for relative motion near perturbed, elliptic orbits. Journal of Celestial Mechanics and Dynamical Astronomy, 97:101-129, 2007.

[20] B. D. Tapley, S. Bettadpur, M. Watkins, and C. Reigber. The gravity recovery and climate experiment: Mission overview and early results. Geophys. Res. Lett., 31(9), May 2004. L09607. 
[21] P. Tempesta, P. Winternitz, J. Harnad, Jr. W. Miller, G. Pogosyan, and M. Rodriguez. CRM, Proceedings \& Lecture Notes, volume 34. Centre de Recherches Mathématiques, Université de Montréal, 2004.

[22] J. Tschauner and P. Hempel. Optimale beschleunigeungsprogramme fur das rendezvousmanover. Acta Astronautica, 10:296-307, 1964.

[23] S.S. Vaddi, S.R. Vadali, and K.T. Alfriend. Formation flying: Accommodating nonlinearity and eccentricity perturbations. Journal of Guidance, Control, and Dynamics, 26(2):214-223, 2003.

[24] W. E. Wiesel and D. J. Pohlen. Canonical Floquet theory. Journal of Celestial Mechanics and Dynamical Astronomy, 58(1):81-96, 1994.

[25] W.E. Wiesel. Relative satellite motion about an oblate planet. Journal of Guidance, Control, and Dynamics, 25(4):776-785, 2002.

[26] W. Xiang and J. L. Jørgensen. Formation flying: A subject being fast unfolding in space. In $5^{\text {th }}$ IAA Symposium on Small Satellites for Earth Observation, Berlin, Germany, 2005. IAA-B5-0309P. 